\title{
Relative contribution of individual versus combined functional imaging studies in predicting seizure freedom in pediatric epilepsy surgery: an area under the curve analysis
}

\author{
Pongkiat Kankirawatana, MD, ${ }^{1}$ Ismail S. Mohamed, MD, ${ }^{1}$ Jason Lauer, MD, ${ }^{2}$ Inmaculada Aban, PhD, ${ }^{3}$ \\ Hyunmi Kim, MD, PhD, MPH, ${ }^{4}$ Rong Li, MD, ${ }^{5}$ Allan Harrison, AS, ${ }^{1}$ Monisha Goyal, MD, ${ }^{1}$ \\ Curtis J. Rozzelle, MD, ${ }^{6}$ Robert Knowlton, MD, MSPH, ${ }^{7}$ and Jeffrey P. Blount, MD ${ }^{6}$ \\ 'Division of Pediatric Neurology, Department of Pediatrics, The University of Alabama at Birmingham School of Medicine, \\ Birmingham, Alabama; ${ }^{2}$ Department of Radiology, Massachusetts General Hospital, Boston, Massachusetts; ${ }^{3}$ Division of \\ Biostatistics, UAB School of Public Health, The University of Alabama at Birmingham, Alabama; ${ }^{4}$ Division of Child Neurology, \\ Department of Neurology and Neurological Sciences, Stanford University School of Medicine, Palo Alto, California; ${ }^{5}$ Department \\ of Pathology, The University of Alabama at Birmingham, Alabama; ${ }^{6}$ Division of Pediatric Neurosurgery, Department of \\ Neurosurgery, The University of Alabama at Birmingham School of Medicine, Birmingham, Alabama; and 'Department of \\ Neurology, University of California, San Francisco, California
}

OBJECTIVE The goal of this study was to evaluate the predictive value and relative contribution of noninvasive presurgical functional imaging modalities based on the authors' institutional experience in pursuing seizure-free surgical outcomes in children with medically refractory epilepsy.

METHODS This was a retrospective, single-institution, observational cohort study of pediatric patients who underwent evaluation and surgical treatment for medically refractory partial epilepsy between December 2003 and June 2016. During this interval, 108 children with medically refractory partial epilepsy underwent evaluation for localization and resective epilepsy surgery. Different noninvasive functional imaging modalities, including ictal SPECT, FDG-PET, and magnetoencephalography-magnetic source imaging, were utilized to augment a standardized paradigm (electroencephalography/ semiology, MRI, and neuropsychology findings) for localization. Outcomes were evaluated at a minimum of 2 years (mean 7.5 years) utilizing area under the receiver operating characteristic curve analysis. Localizing modalities and other clinical covariates were examined in relation to long-term surgical outcomes.

RESULTS There was variation in the contribution of each test, and no single presurgical workup modality could singularly and reliably predict a seizure-free outcome. However, concordance of presurgical modalities yielded a high predictive value. No difference in long-term outcomes between inconclusive (normal or diffusely abnormal) and abnormal focal $\mathrm{MRI}$ results were found. Long-term survival analyses revealed a statistically significant association between seizure freedom and patients with focal ictal EEG, early surgical intervention, and no history of generalized convulsions.

CONCLUSIONS Comprehensive preoperative evaluation utilizing multiple noninvasive functional imaging modalities is not redundant and can improve pediatric epilepsy surgical outcomes.

https://thejns.org/doi/abs/10.3171/2020.1.FOCUS19974

KEYWORDS pediatric epilepsy; epilepsy surgery; outcome; presurgical workup

$\mathrm{T}$ HE benefit of surgery for patients with medically resistant epilepsy is well established. ${ }^{31,32}$ However, extratemporal, neocortical epilepsy predominates in children and is historically difficult to localize.,11,21 As a result, more extensive investigation is often needed, and surgical outcomes are more variable. Children with normal MRI findings are conventionally less likely to be considered good candidates for epilepsy surgery due to the likelihood of modest surgical outcome. ${ }^{20,22,26}$

The cornerstone of localization studies at virtually all

ABBREVIATIONS AUC = area under the ROC curve; EEG = electroencephalography; MEG-MSI = magnetoencephalography-magnetic source imaging; PMC = patient management conference; $\mathrm{ROC}=$ receiver operating characteristic.

SUBMITTED December 1, 2019. ACCEPTED January 28, 2020.

INCLUDE WHEN CITING DOI: 10.3171/2020.1.FOCUS19974. 
centers include video-electroencephalography (EEG) and MRI. These standard, indispensable initial investigations in presurgical epilepsy evaluation enable characterization of laterality, lobar focality, and semiology. Morefocal localization is typically accomplished via the use of functional imaging tests. Significant advancements in multimodality neuroimaging include brain FDG-PET scanning, ${ }^{5}$ ictal blood flow studies assessed by SISCOM (subtraction ictal SPECT co-registered to MRI) or ictal SPECT, 3,15,23,25,27 and magnetoencephalography-magnetic source imaging (MEG-MSI). ${ }^{15,17}$

Due to cost and provider practice preference, there are significant disparities between centers with regard to the use of functional imaging modalities. There are no clear comparative data demonstrating superiority of one modality over another. Furthermore, there is limited information available about the utility of a single localization study versus a limited battery of studies to attain sufficient accuracy of localization to optimize the likelihood of postoperative seizure freedom. Third-party payers are sometimes reluctant to underwrite more than one test that serves the same localization purpose. Furthermore, each of the functional imaging studies involves significant institutional cost commitment, so there is a fiscal incentive to determine the most efficient and cost-effective algorithm of studies. Despite robust literature advocating utility of specific functional imaging modalities, there are limited comparisons of presurgical investigations alone or in combination to select the candidates who are most likely to benefit from epilepsy surgery. The goal of this study was to explore the relative contribution and prognostic capability of each imaging modality utilized at our center in predicting seizure freedom after pediatric epilepsy surgery.

\section{Methods}

\section{Cohort and Definitions}

Patients who underwent epilepsy surgery at Children's of Alabama between December 2003 and June 2016 were identified in our prospectively maintained pediatric epilepsy surgery database. We excluded patients who had less than 2 years of follow-up and those who underwent functional hemispherectomy or vagus nerve stimulation device implantation. The duration of epilepsy was stratified at 60 months based on published standards of long-term outcome analysis in pediatric epilepsy surgery analysis. ${ }^{30}$ Demographics of the cohort are presented in Table 1.

\section{Preoperative Epilepsy Surgery Evaluation}

All patients received a standard presurgical evaluation, including a detailed clinical history, seizure history (including semiology, seizure frequency, and epilepsy duration), prolonged video-EEG monitoring (with scalp electrodes placed according to the International 10-20 system), and brain MRI. Other presurgical investigations, including FDG-PET, ictal SPECT, and MEG-MSI, were performed in selected patients as clinically indicated by the judgment of the attending epileptologist or augmented by the consensus of the patient management conference (PMC). Most patients received a battery of more than one investigation, but there was no firm protocol to direct
TABLE 1. Demographic data

\begin{tabular}{|c|c|}
\hline & Value \\
\hline Sex, M/F & $65: 43$ \\
\hline Mean age at seizure onset (range) & $4.2 \pm 4.05$ yrs ( 2 wks -16 yrs $)$ \\
\hline Mean age at op (range) & $10.2 \pm 5.1 \mathrm{yrs}(4 \mathrm{mos}-20.8 \mathrm{yrs})$ \\
\hline \multicolumn{2}{|l|}{ Side of op } \\
\hline Rt & 59 \\
\hline $\mathrm{Lt}$ & 47 \\
\hline Bilat & 1 (patient w/ tuberous sclerosis) \\
\hline Invasive EEG or icEEG* & 80 \\
\hline No icEEG (lesionectomy) & 28 \\
\hline \multicolumn{2}{|l|}{ Lobe } \\
\hline Frontal & 35 \\
\hline Temporal & 18 \\
\hline Parietal & 10 \\
\hline Occipital & 3 \\
\hline Multilobar & 42 \\
\hline \multicolumn{2}{|l|}{ Etiology } \\
\hline Cortical dysplasia† & 85 \\
\hline $\begin{array}{l}\text { Low-grade tumor } \ddagger \text { (ganglioglioma, } \\
\text { oligodendroglioma, etc.) }\end{array}$ & 9 \\
\hline Hippocampal sclerosis & 2 \\
\hline Other§ & 9 \\
\hline $\begin{array}{l}\text { No tissue available/no pathologi- } \\
\text { cal diagnosis }\end{array}$ & 3 \\
\hline $\begin{array}{l}\text { Mean duration of postop follow-up } \\
\text { (range), yrs }\end{array}$ & $7.55 \pm 2.97(2-16)$ \\
\hline
\end{tabular}

icEEG = intracranial EEG.

Values represent the number of patients (\%) unless stated otherwise. Mean

values are presented as mean \pm SD.

* Includes subdural grids, strips, and depth electrodes.

$\dagger$ Includes 12 cases of tuberous sclerosis.

$\ddagger$ e.g., ganglioglioma, oligodendroglioma.

$\S$ e.g., gliosis, trauma, intraventricular hemorrhage.

decisions about functional imaging. Like most centers, important presurgical investigation data, including interictal EEG, ictal EEG, MRI, FDG-PET, ictal SPECT, and MEG-MSI findings were discussed and considered during our PMC. Each of the results of these investigations were independently classified into 3 main categories: localized (lobar distribution); lateralized (hemispheric); and nonlocalizing, including diffuse, multifocal, or nonlateralized results.

To define the relative contribution of each study, we sought to perform logistic analysis of the 6 presurgical investigations (interictal EEG, ictal EEG, MRI, FDGPET, ictal SPECT, and MEG-MSI) with regard to surgical outcome. This required that we measure the localizing contribution of each study. We chose to define categorical variables in which each study result was retrospectively classified into one of 3 categories that reflected the study result with regard to the defined surgical target. We defined each score as follows: 0, normal findings or abnormal MRI but inconclusive findings (diffusely abnormal or 
multifocal lesions); 1, abnormal lateralized lesion to the side of the surgery; and 2, abnormal localized lesion, concordant with the site of epilepsy surgery. This scoring system was applied across all 6 covariates (interictal EEG, ictal-EEG, MRI, FDG-PET, ictal SPECT, and MEG-MSI).

\section{MRI}

All patients underwent MRI with an epilepsy protocol that included diffusion images, axial T2-weighted images, coronal T1-weighted inversion recovery images, coronal FLAIR images and a 3D T1-weighted image. Prior to 2010, MRI scans were obtained using a 1.5T scanner; since then, all MRI scans have been obtained using a 3T scanner. All MRI scans were reviewed by neuroradiologists and experienced epileptologists independently and corroborated during the PMC. Similar to EEG results, the MRI results were retrospectively classified according to the consensus of the PMC and with respect to the location of surgery as stated above. We included patients with normal MRI or abnormal inconclusive, diffuse, or multifocal MRI findings in the same group (0) reflective of the fact that MRI did not produce sufficient localizing information to influence surgical decision-making.

\section{FDG-PET}

FDG-PET scans were obtained in selected patients on an outpatient basis. A 5- to 10-mCi injection of FDG was performed during the interictal state, with the patient resting with his or her eyes closed or, in cases of uncooperative children, under sedation. Simultaneous EEG recording was also performed during injection of the tracer to assist in the interpretation of the results and ensure that no seizures or excessive interictal discharges occurred at the time of the injection. Visual analysis of FDG-PET scans, coregistered to the patient's MRI study was performed by reviewers with expertise in the interpretation of FDGPET scans and independent of other imaging studies. After 2010, FDG-PET image coregistration with MRI was performed using MIM (MIM Software Inc.). A record of group consensus interpretation was achieved with regard to presence and localization of relative focal hypometabolism, and then the results were classified as outlined above with consideration to the side of surgery based on the PMC consensus.

\section{Ictal SPECT}

Ictal SPECT was attempted in all patients with normal or nonlateralized MRI results. Ictal SPECT was also attempted in select MRI-lateralized cases where other presurgical evaluations revealed inconclusive findings or findings incongruent with the MRI findings. This decision was made by group consensus at the epilepsy surgery case conference. Ictal and interictal SPECT scans were performed with injections of $20-40 \mathrm{mCi}$ of ${ }^{99 \mathrm{~m}} \mathrm{Tc}$ hexamethylpropyleneamine-oxime ( ${ }^{99 \mathrm{~m}} \mathrm{Tc}$ HMPAO). Scans were acquired within 1 to 3 hours of injection in all cases. Voxel difference values considered significant were based on the criterion that they exceed 2 standard deviations of the variance computed for all voxels in the difference image. The results were retrospectively classified with respect to PMC localization consensus as outlined above.

\section{MEG-MSI}

MEG-MSI was performed in most patients with normal or nonlateralized MRI and some selected MRI-positive cases when other presurgical evaluations revealed inconclusive findings or findings incongruent with the MRI findings. Between 2003 and 2006, MSI was performed in most of the cases under an NIH NINDS grant (no. K23 NS02218 to R.K.). After 2007, magnetic source images were obtained in select cases or based on the group consensus at the PMC. Because insurance coverage was variable, not all patients underwent MEG-MSI. Continuous recordings of spontaneous cerebral activity were recorded using a whole-head, 148-channel biomagnetometer system (4D Neuroimaging). MEG-MSI data were analyzed using an equivalent current dipole model. MEG-MSI results were classified with respect to localization as outlined above.

\section{Seizure Outcomes}

Seizure outcomes were classified using Engel classification: class I, free of disabling seizures; class II, rare disabling seizures; class III, worthwhile improvement; and class IV, no worthwhile improvement. ${ }^{8,29}$ Each patient was followed periodically at 1-, 3-, 6-, and serial 12-month intervals after surgery. If the patient's last follow-up was longer than 12 months at the time of closing the study for statistical analysis, a telephone follow-up was made by either an epilepsy clinical nurse practitioner or pediatric epileptologist to verify the seizure outcome status.

\section{Statistical Analysis}

\section{Postoperative Short-Term Surgical Outcomes}

We defined the short-term surgical outcome as the outcome 1 year after surgery and classified the patients into two groups: completely seizure free (Engel class I) and not seizure free (Engel classes II-IV). Logistic regression analysis was used to model the short-term surgical outcomes and the prognostic capacity of each of the 6 presurgical investigations (interictal EEG, ictal EEG, MRI, FDG-PET, ictal SPECT, and MEG-MSI) to target the surgical investigation and subsequent resection. We assessed the predictive ability using the area under the receiver operating characteristic (ROC) curve (AUC), which evaluates the sensitivity and specificity of the model. ROC association statistics of each variable (MRI, ictal EEG, interictal EEG, FDG-PET, ictal SPECT, and MEG-MSI) was performed both individually and sequentially combined to compare the predictive value of each variable based on the AUC. Since not all patients underwent all investigations, logistic regression analyses were separately performed for each individual investigation model and all investigations.

In the next step, we tried to develop an algorithm to guide the use of different presurgical modalities that also can be used to predict seizure freedom. We created a model, with the base model being MRI. We built on this model by adding other modalities to examine how having additional presurgical modalities could improve the model. We calculated the Akaike information criterion, which is an index of model fit. 


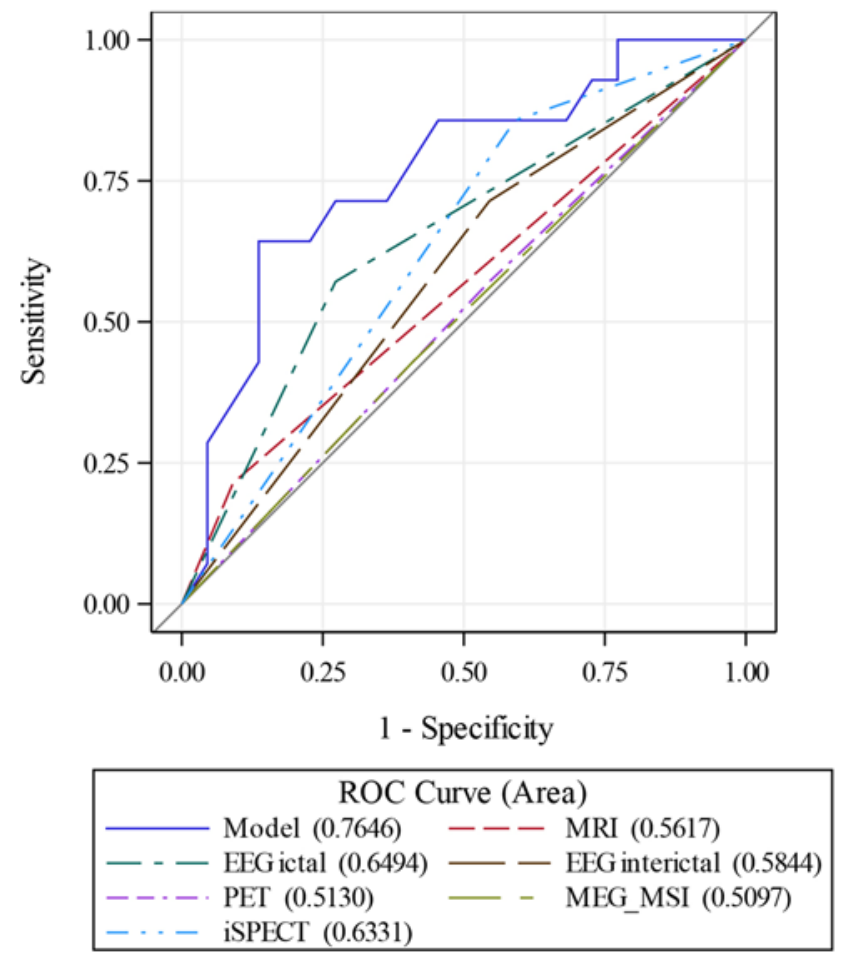

FIG. 1. ROC association statistics of each variable (MRI, ictal EEG, interictal EEG, MEG-MSI, FDG-PET, and ictal SPECT [iSPECT]) performed individually. None of the ROC individual model results were statistically significant in predicting the short-term surgical outcomes. AUC values: MRI, $0.5617(n=108)$; ictal EEG, $0.6494(n=108)$; interictal EEG, $0.5844(n=108) ;$ MEG-MSI, $0.5097(n=51)$; FDG-PET, $0.5130(n$ $=92)$; and ictal SPECT, $0.6331(n=75)$.

\section{Postoperative Long-Term Outcomes}

We defined the longest available clinic visit (2-year minimum) as the long-term outcome. As detailed in Table 1 , the mean long-term follow-up was 7.5 years. We analyzed these outcomes using survival analysis, in particular, Kaplan-Meier curves and the Cox proportional hazards model. Kaplan-Meier curves for each variable were stratified into a scoring system $(0$, normal/nonspecific; 1 , abnormal and lateralized to the hemisphere in relation to the side of surgery; and 2, abnormal lobar distribution, concordant to the location of surgery).

Statistical significance was tested using the log-rank test and comparison of $95 \%$ confidence intervals. We also examined the role of covariates, including sex, race, age at operation, age at onset, and operation side. Statistical significance was determined at $\mathrm{p}<0.05$.

\section{Results}

\section{Patient Population}

A total of 108 patients who underwent resective epilepsy surgery for medically refractory epilepsy and fulfilled all inclusion and exclusion criteria were included in this study. Patient age at seizure onset ranged from 2 weeks to 16 years (mean $4.2 \pm 4.05$ years $[ \pm \mathrm{SD}]$ ). The age at the first operation ranged from 4 months to 20.8 years (mean
$10.2 \pm 5.1$ years). The follow-up duration ranged from 2 to 16 years (mean $7.55 \pm 2.97$ years). Normal MRI findings were seen in 29 patients, whereas the MRI findings were abnormal in the remaining 79 patients. Among the group with normal MRI findings, 9 of 29 patients were seizure free at 12 months; in the MRI abnormal group, 40 of 79 patients were seizure free at the 12-month follow-up.

\section{Presurgical Evaluation}

MRI and video-EEG were performed in all patients. Other investigations were performed according to the preference of the attending epileptologist, which was augmented by consensus from our weekly PMC during the phase 1 presurgical evaluation. Only 36 patients in this cohort had undergone all investigations consisting of MRI, video-EEG, FDG-PET, ictal SPECT, and MEGMSI. FDG-PET scanning of the brain was performed in 92 patients, 75 patients had ictal SPECT, and 51 patients had MEG-MSI studies. Twenty-one patients with normal MRI findings had all 3 presurgical modalities performed (FDG-PET, MEG-MSI, and ictal SPECT).

\section{Postoperative Outcome}

\section{ROC Curve Comparison: Short-Term Outcome}

None of the ROC individual-model results was successful in predicting short-term seizure freedom. For full-model analysis, we included the 36 patients who underwent all investigations (MRI, video-EEG, MEG-MSI, FDG-PET, and ictal SPECT). Similarly, no single modality alone was successful in predicting seizure freedom (Engel class I) at the 1-year follow-up (Fig. 1). We then tested the model in the MRI-negative or nonlocalizing group only $(n=21)$. None of the presurgical modalities alone was predictive of seizure freedom at 1 year; however, concordant localized results of all 3 presurgical modalities were highly predictive of seizure freedom at the 1-year follow-up (specificity $93 \%$ and sensitivity $33 \%$, Table 2).

\section{Long-Term Seizure Outcome}

Localized ictal EEG was the only variable predictive of long-term good surgical outcome ( $p=0.0031$; Fig. 2B). All other individual variables (interictal EEG, MRI, FDGPET, ictal SPECT, and MEG-MSI) did not yield any statistically significant difference in Kaplan-Meier analysis (Fig. 2A and C-F.)

\section{Effect of Other Clinical Variables on Long-Term Outcomes}

Comparison of Kaplan-Meier curves of other clinical variables, including duration of epilepsy prior to operation (within 60 months of seizure onset vs after 60 months of seizure onset), utilization of invasive EEG (with subdural grids vs without subdural grids), history of secondarily generalized convulsive seizures (presence vs absence of generalized convulsive seizures), and an abnormal pathological finding of balloon cells (presence vs absence of balloon cells), were analyzed using the log-rank test and comparison of $95 \%$ confidence intervals. Only two clinical variables, early epilepsy surgery (within 60 months of seizure onset, $\mathrm{p}=0.0098$ ) and the absence history of secondarily generalized convulsive seizures, were statisti- 
TABLE 2. Sensitivity and specificity for different presurgical modalities against long-term seizure freedom and concordant localized test results in all 3 presurgical modalities in patients with normal or nonlocalizing MRI findings

\begin{tabular}{|c|c|c|c|c|c|c|}
\hline & Rule & AUC & Index & Estimate & 95\% Lower Limit & 95\% Upper Limit \\
\hline \multirow{6}{*}{$\begin{array}{l}\text { MEG-MSI, ISPECT } \\
\text { \&/or FDG-PET w/ } \\
\text { results localized }\end{array}$} & \multirow{2}{*}{$\geq 1$ modality } & \multirow{2}{*}{0.51667} & Specificity & 0.13333 & 0.01658 & 0.4046 \\
\hline & & & Sensitivity & 0.83333 & 0.35877 & 0.99579 \\
\hline & \multirow{2}{*}{$\geq 2$ modalities } & \multirow{2}{*}{0.56667} & Specificity & 0.53333 & 0.26586 & 0.78733 \\
\hline & & & Sensitivity & 0.33333 & 0.04327 & 0.77722 \\
\hline & \multirow{2}{*}{3 modalities } & \multirow{2}{*}{0.63333} & Specificity & 0.93333 & 0.68052 & 0.99831 \\
\hline & & & Sensitivity & 0.33333 & 0.04327 & 0.77722 \\
\hline \multirow{2}{*}{$\begin{array}{l}\text { MEG-MSI results } \\
\text { localized }\end{array}$} & \multirow{2}{*}{ MEG-MSI only } & \multirow{2}{*}{0.56667} & Specificity & 0.53333 & 0.26586 & 0.78733 \\
\hline & & & Sensitivity & 0.33333 & 0.04327 & 0.77722 \\
\hline \multirow{2}{*}{$\begin{array}{l}\text { iSPECT results } \\
\text { localized }\end{array}$} & \multirow{2}{*}{ iSPECT only } & \multirow{2}{*}{0.61667} & Specificity & 0.4 & 0.16336 & 0.67713 \\
\hline & & & Sensitivity & 0.83333 & 0.35877 & 0.99579 \\
\hline \multirow{2}{*}{$\begin{array}{l}\text { FDG-PET results } \\
\text { localized }\end{array}$} & \multirow{2}{*}{ FDG-PET only } & \multirow{2}{*}{0.61667} & Specificity & 0.66667 & 0.3838 & 0.88176 \\
\hline & & & Sensitivity & 0.33333 & 0.04327 & 0.77722 \\
\hline
\end{tabular}

iSPECT $=$ ictal SPECT.

Concordant localized had the highest specificity in predicting long-term seizure freedom.

cally significant in predicting long-term seizure-free outcomes $(\mathrm{p}=0.0193$; Fig. 3$)$.

\section{Discussion}

Selection of patients for epilepsy surgery is a complex decision-making process and depends on the concordance between clinical data and different modalities used in the presurgical workup. MRI and video-EEG are critical, and both play an important role in the decisionmaking process. ${ }^{5,27,28}$ Other functional imaging studies are utilized either individually or in combination to support the decision-making process. ${ }^{2-4,6,16-18,23}$ However, few centers have all these presurgical investigations available. Hence, the process of presurgical investigation is usually dependent on the availability of different modalities in each center as well as individual provider preference and experience. ${ }^{8,13}$ In this study, we aimed to review our experience to help identify which of the noninvasive functional imaging tests we used had the best ability to regionally inform the surgical investigation and result in the best surgical outcome.

Ultimately, the surgical result arises from the accuracy of defining the region of seizure onset or disrupting a critical network essential for the propagation of the seizure.,12 Neocortical epilepsy predominates in children and is difficult to localize. ${ }^{7,8,12}$ The neocortex has robust projections, which make rapid and diffuse spread of epileptiform discharges common. The characteristic pathologic substrate is cortical dysplasia, which is often MRI occult. ${ }^{17,10,12,19 \text {, }}$ 24,33 Staged procedures in which a noninvasive evaluation (stage I) informs placement of intracranial electrodes (stage II) are common. Results of intracranial EEG findings from invasive electrodes allow confirmation of the precise region of ictal onset and propagation and the critical relationships with adjacent eloquent cortex.12,18 Surgical success or failure is dependent on the accuracy of the entire process as well as the complexity of the pathological network and its inherent propensity for reorganization and recurrence. ${ }^{10-12,14,19,24}$ As such, it is unlikely that any single factor or test is capable of informing the entirety of surgical success. However, the practical issue remains as to the choice of functional imaging modalities. This study takes a large clinical experience with a prolonged followup time to address the potential capability of commonly available and widely utilized noninvasive functional imaging studies to predict good surgical outcome. An ROC curve analysis was chosen due to its capability to provide information about the benefit of a given investigation. The AUC in ROC curve analysis provides an assessment of the utility of a given test.

Functional imaging contributes to the initial stage of localization to corroborate early findings in semiology, EEG, and MRI. ${ }^{13,23,27}$ Stage II intracranial EEG investigations characteristically closely refine the focus of the resection and avoid injury to eloquent tissue but rarely fundamentally contribute to lobar localization or lateralization. Quantification of the role to which functional imaging defines placement of intracranial electrodes is elusive, and no standardized metric for this analysis has been reported. Our group, like many others, uses a standardized and practical approach in which functional imaging is used to corroborate EEG, semiology, and MRI findings in an individually tailored manner. Regions of interest are defined at conference by consensus and are covered broadly at surgery with sufficient margin so as to define borders of hyperexcitability. The focused question here pertains to the capacity of each noninvasive element of the preoperative evaluation to predict and inform good surgical outcome as part of a standardized overall surgical paradigm. For this reason, the contribution of stage II electrodes was not incorporated in this analysis. Stage II electrodes refine the margins and define the extent of the resection but do not primarily and initially inform the targeted regions of brain under investigation.

Classification of the preoperative tests was designed to be practical and straightforward. Normal and widely diffuse onset was combined because neither is contributory 

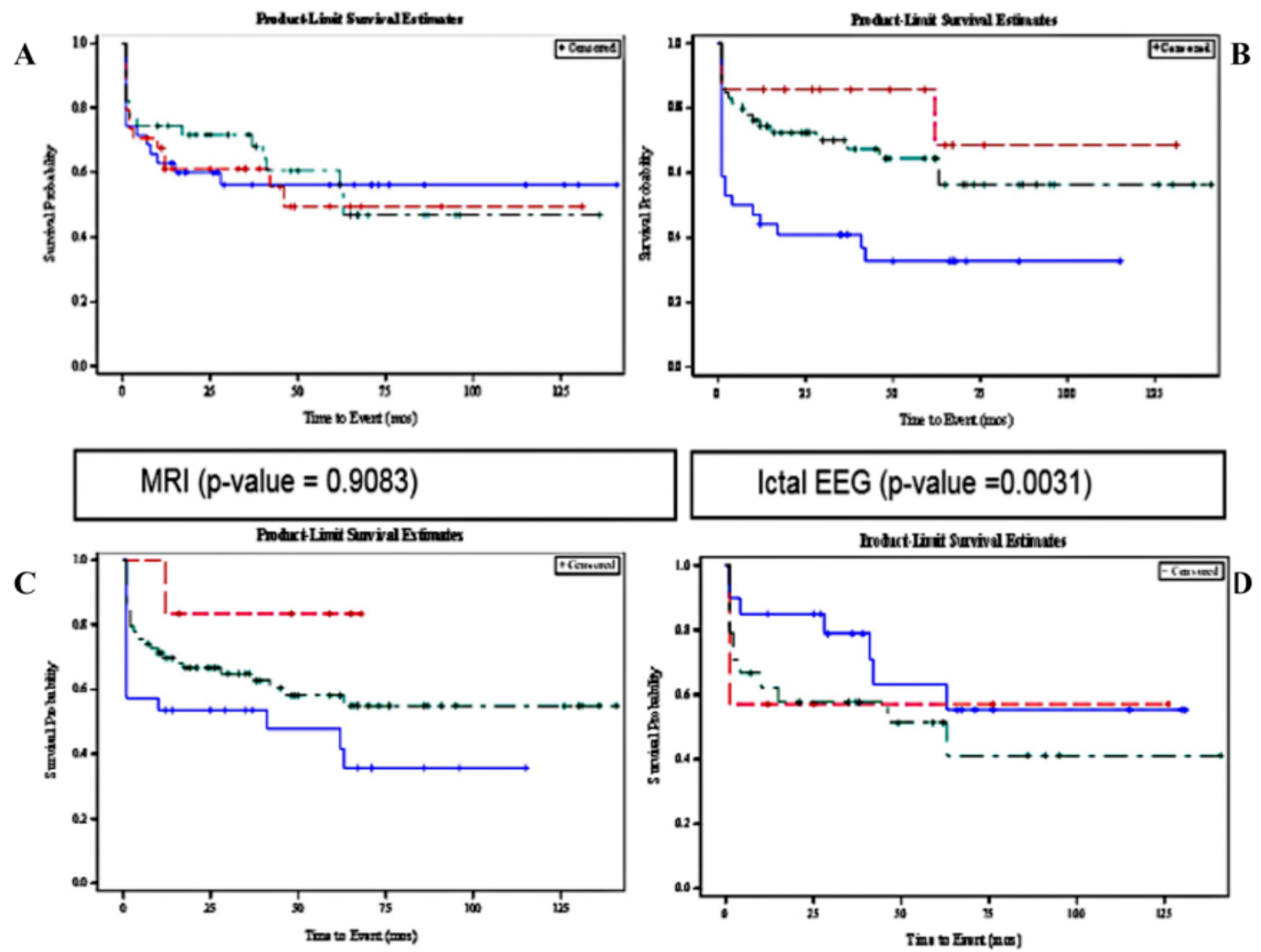

Interictal EEG (p-value $=0.1025)$

MEG (p-value $=0.4981)$

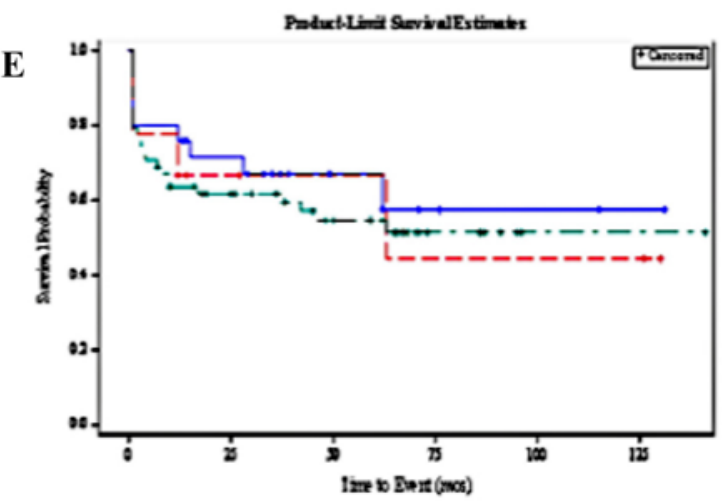

FDG-PET ( $p$-value $=0.7983$ )

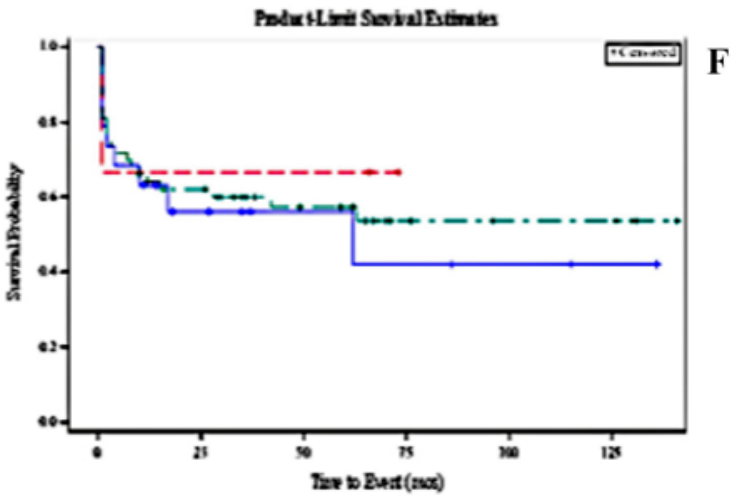

iSPECT $(\mathrm{p}$-value $=0.8336$ )

$0--\cdot 1-\cdots 2$

FIG. 2. Kaplan-Meier curves for each variable were stratified into a scoring system of 0 , normal/nonspecific; 1 , abnormal and lateralized to the hemisphere in relation to the side of surgery; and 2, abnormal lobar distribution, concordant to the location of surgery and then compared. Correlation with good surgical outcome was found only in ictal EEG $(p=0.0031)$.

to focal localization of partial epilepsy. It is recognized that these EEG signatures arise from different processes, but, from a practical localization perspective, they lack localizing value and thus were combined. Grade I indicated adequate lateralizing data to confirm laterality, and grade
II refined a lobar region where subsequent resection ensued. It is also recognized that significant refinement to the resection occurs from the results of intracranial EEG but that accurate lobar localization is the essential objective of the noninvasive investigations. 

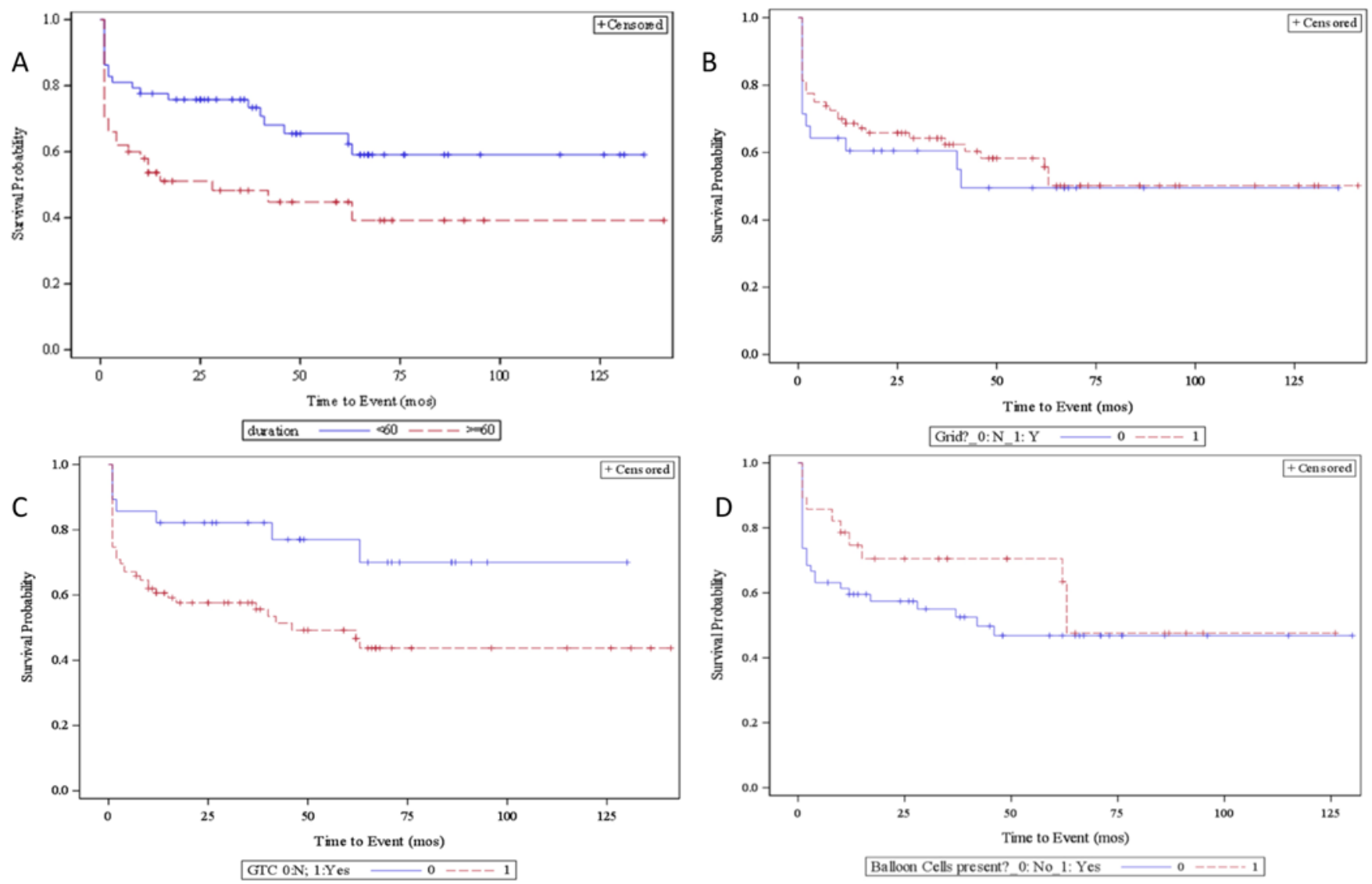

FIG. 3. Kaplan-Meier curves of different clinical variables. A: Duration of epilepsy prior to operation (<60 months vs $>60$ months). The group that underwent epilepsy surgery earlier (within 60 months after seizure onset) had significantly better long-term outcomes than the group with delayed surgery (> 60 months after seizure onset) $(p=0.0098)$. B: Surgery with invasive EEG versus without $(p=0.8336)$. C: Patients with and without a history of generalized convulsive seizures. Patients without a history of generalized convulsive seizures had significantly better long-term outcome $(p=0.0193)$. GTC = generalized tonic-clonic. D: Presence or absence of balloon cells. The presence of balloon cells seems to be associated with a better long-term outcome, but it did not reach statistical significance $(p=0.2822)$. Relapse of seizure freedom with focal cortical dysplasia type $2 B$ is likely due to incomplete resection, which occurred in 18 patients. This was uniformly due to colocalization with eloquent tissue and a reluctance to knowingly impart neurological injury.

\section{Predictors of Short-Term Seizure Freedom}

None of the presurgical modalities alone was predictive of short-term (12-month) seizure freedom. However, concordance of multiple presurgical modalities yielded the highest predictive value (AUC $=0.7646$ ). In the MRInegative group, concordant localized results in all 3 presurgical modalities was highly specific (specificity 93\%) in predicting seizure freedom at 12 months. Figure 4 illustrates how the full utilization of presurgical noninvasive multimodalities help with placement of invasive intracranial electrodes. The question of outcome 1 year after epilepsy surgery is rarely addressed separately in the literature but can be inferred from studies with a relatively short duration of postsurgical follow-up. The most likely reason for recurrent seizures shortly after epilepsy surgery is incomplete resection of the epileptogenic lesion or zone. . $^{6,19}$ Incomplete resections can occur as a result of 1) overlap of the epileptogenic zone with eloquent cortex; 2) an extensive epileptogenic zone beyond visible imaging changes in lesional epilepsy; and 3) inadequate sampling of the epi- leptogenic network during invasive monitoring secondary to imprecise presurgical workup. ${ }^{19}$ Hence, a single localized presurgical workup modality might not be predictive of short-term surgical seizure freedom in patients with normal or nonlocalizing MRI. Concordance of different presurgical workup modalities is more predictive, as it allows better selection of patients and adequate estimation of the spatial extent of the epileptogenic zone and more targeted invasive monitoring.

\section{Presurgical Workup in Pediatric Epilepsy: Less Is Not Always More}

An important question in pediatric epilepsy surgery evaluation is how many investigations are needed and whether these presurgical investigations should be acquired sequentially or together to achieve the highest predictive ability in the most cost-effective fashion. In order to tackle this, we examined an algorithm with the base model has a normal MRI and then added each variable into the base model. No single modality was successful in 

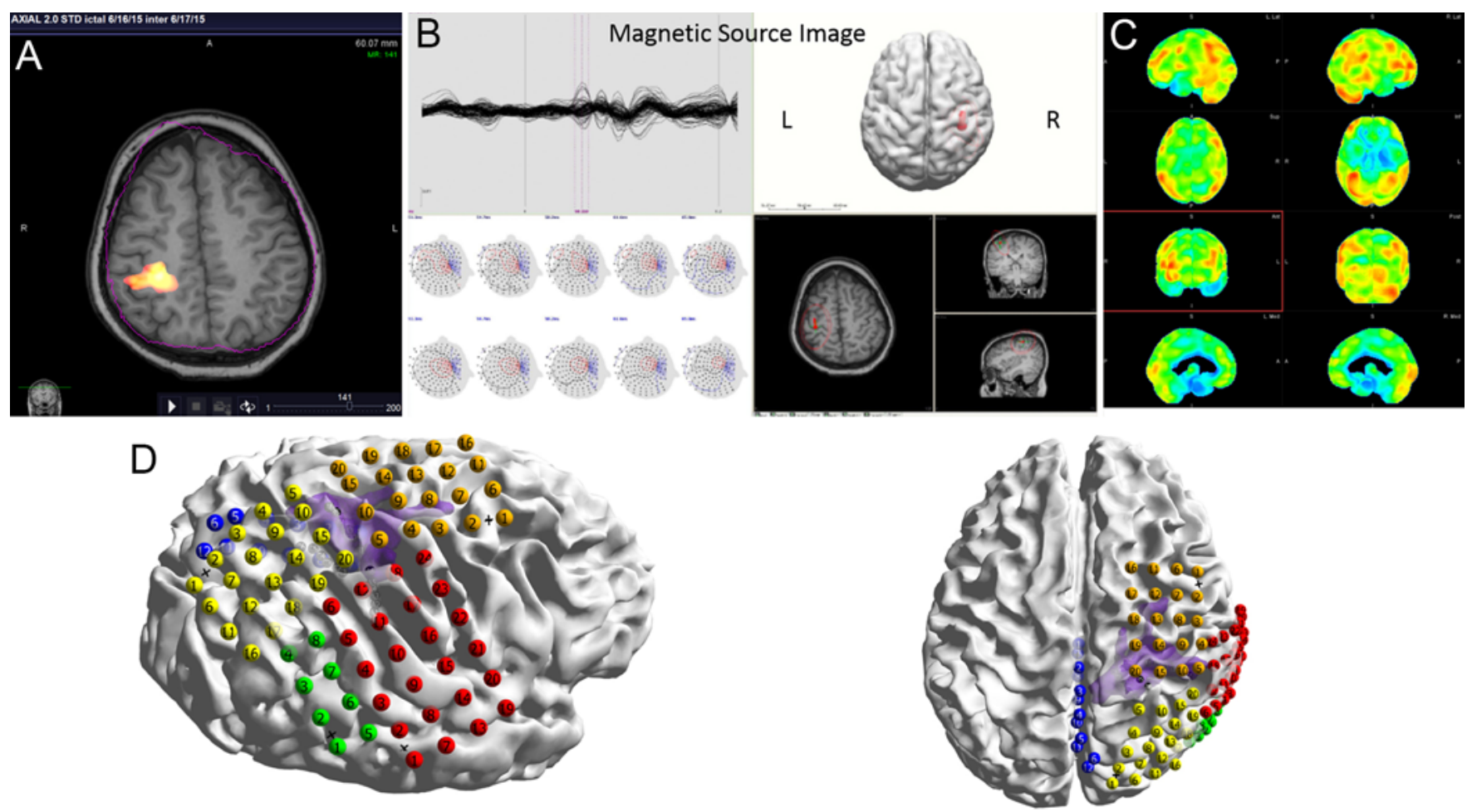

FIG. 4. Illustrative case of full utilization of all noninvasive presurgical workup modalities in assisting invasive electrodes (subdural and depth electrodes) placement in an 11-year-old boy with medically refractory focal epilepsy and completely normal 3T MRI findings. A: Localized increased blood flow on ictal SPECT. B: Localized MEG-MSI. C: Questionable slightly hypometabolic FDG-PET uptake at the right parietal. All modalities are concordant to the right parietal lobe. These data were then coregistered with the preoperative MRI of the brain navigator system. D: Location of the subdural and depth electrode placement. Ictal SPECT (lavender) and MEG data are coregistered into the patient's 3D MRI image. This patient became completely seizure free after right parietal resection.

predicting postoperative seizure freedom. However, concordant results from all 3 presurgical modalities (FDGPET, ictal SPECT, and MEG-MSI) were highly specific in predicting seizure freedom.

\section{Study Limitations}

This study does not investigate the role of stage II invasive electrodes in seizure outcome. Rather, it focuses on the contribution of noninvasive functional imaging to attain lateralization or lobar localization and thus guide either resection in the setting of an MRI abnormality or implantation of electrodes in cases of normal findings on MRI. Lobar localization is the desired objective for staged cases, and functional imaging serves to guide and inform selected regions and lobes for electrode placement. The relative contribution of imaging to guide placement of electrodes is elusive to quantify and is accomplished in a practical standardized fashion shared by most surgical epilepsy teams. A region of interest is defined by noninvasive studies and refined by discussion at conference. Sufficient safe coverage is accomplished at surgery to define margins of hyperexcitability and relationships with adjacent eloquent cortex.

Not all patients received all modalities of presurgical investigations. This is due to differences in provider preference and health insurance coverage. We included patients with normal MRI or abnormal inconclusive findings or diffuse or multifocal MRI findings in the same group, reflective of the fact that MRI does not result in sufficient localizing information that will influence clinical decision-making. Patients were not stratified by age, and there are some indications of relatively better contributions for semiology in age-specific subcohorts. While these issues are not ideal from a study design perspective, they accurately reflect a typical cohort at most pediatric epilepsy referral centers. The retrospective nature of the study would make it subject to variability in clinical practice over time.

\section{Conclusions}

A large pediatric surgical cohort with extended followup was reviewed with regard to the utility and relative contribution of commonly utilized functional imaging tests. No single test reliably predicted long-term seizure freedom. The contribution of invasive electrodes to refine the resection cannot be determined from this analysis. The best predictor of long-term seizure freedom was concordant ictal EEG. However, the results of this study underline the importance of the central concept of study concordance. When more than 3 tests implicated the same lobe of ictal onset, the positive predictive value for resective surgery in that lobe exceeded $93 \%$. As such, the 
utilization of multiple presurgical investigations is not inherently redundant and could improve surgical outcome in pediatric focal epilepsy with normal or nonlocalizing MRI.

\section{Acknowledgments}

We gratefully acknowledge the assistance of Jon Betts, Jennifer Glen, Sula Gillespie, Jennifer Williams, Raven O'Leary, Pam Parks, Pam Miller, Sarah Kiel, Tracy Cartwright, Cathy Lee, Allyson Baker, Jeff Killen, and Trei King.

Some of the MEG-MSI studies (those obtained between 2003 and 2005) were supported by an NIH NINDS grant (grant no. K23 NS02218 to R.K.).

\section{References}

1. Boonyapisit K, Najm I, Klem G, Ying Z, Burrier C, LaPresto E, et al: Epileptogenicity of focal malformations due to abnormal cortical development: direct electrocorticographichistopathologic correlations. Epilepsia 44:69-76, 2003

2. Carne RP, O'Brien TJ, Kilpatrick CJ, MacGregor LR, Hicks RJ, Murphy MA, et al: MRI-negative PET-positive temporal lobe epilepsy: a distinct surgically remediable syndrome. Brain 127:2276-2285, 2004

3. Cascino GD, Buchhalter JR, Mullan BP, So EL: Ictal SPECT in nonlesional extratemporal epilepsy. Epilepsia 45 (Suppl 4):32-34, 2004

4. Chandra PS, Vaghania G, Bal CS, Tripathi M, Kuruwale N, Arora A, et al: Role of concordance between ictal-subtracted SPECT and PET in predicting long-term outcomes after epilepsy surgery. Epilepsy Res 108:1782-1789, 2014

5. Chapman K, Wyllie E, Najm I, Ruggieri P, Bingaman W, Lüders J, et al: Seizure outcome after epilepsy surgery in patients with normal preoperative MRI. J Neurol Neurosurg Psychiatry 76:710-713, 2005

6. Chugani HT: PET in preoperative evaluation of intractable epilepsy. Pediatr Neurol 9:411-413, 1993

7. Cossu M, Lo Russo G, Francione S, Mai R, Nobili L, Sartori I, et al: Epilepsy surgery in children: results and predictors of outcome on seizures. Epilepsia 49:65-72, 2008

8. Cross JH, Jayakar P, Nordli D, Delalande O, Duchowny M, Wieser HG, et al: Proposed criteria for referral and evaluation of children for epilepsy surgery: recommendations of the Subcommission for Pediatric Epilepsy Surgery. Epilepsia 47:952-959, 2006

9. Engel J Jr, McDermott MP, Wiebe S, Langfitt JT, Stern JM, Dewar S, et al: Early surgical therapy for drug-resistant temporal lobe epilepsy: a randomized trial. JAMA 307:922-930, 2012

10. Englot DJ, Han SJ, Rolston JD, Ivan ME, Kuperman RA, Chang EF, et al: Epilepsy surgery failure in children: a quantitative and qualitative analysis. J Neurosurg Pediatr 14:386-395, 2014

11. Garcia Gracia C, Yardi R, Kattan MW, Nair D, Gupta A, Najm I, et al: Seizure freedom score: a new simple method to predict success of epilepsy surgery. Epilepsia 56:359-365, 2015

12. Hosoyama H, Matsuda K, Mihara T, Usui N, Baba K, Inoue Y, et al: Long-term outcomes of epilepsy surgery in 85 pediatric patients followed up for over 10 years: a retrospective survey. J Neurosurg Pediatr 19:606-615, 2017

13. Jayakar P, Gaillard WD, Tripathi M, Libenson MH, Mathern GW, Cross JH: Diagnostic test utilization in evaluation for resective epilepsy surgery in children. Epilepsia 55:507-518, 2014

14. Jehi L, Yardi R, Chagin K, Tassi L, Russo GL, Worrell G, et al: Development and validation of nomograms to provide individualised predictions of seizure outcomes after epilepsy surgery: a retrospective analysis. Lancet Neurol 14:283-290, 2015

15. Kaiboriboon K, Lowe VJ, Chantarujikapong SI, Hogan RE: The usefulness of subtraction ictal SPECT coregistered to MRI in single- and dual-headed SPECT cameras in partial epilepsy. Epilepsia 43:408-414, 2002

16. Knowlton RC, Elgavish R, Howell J, Blount J, Burneo JG, Faught E, et al: Magnetic source imaging versus intracranial electroencephalogram in epilepsy surgery: a prospective study. Ann Neurol 59:835-842, 2006

17. Knowlton RC, Elgavish RA, Bartolucci A, Ojha B, Limdi N, Blount J, et al: Functional imaging: II. Prediction of epilepsy surgery outcome. Ann Neurol 64:35-41, 2008

18. Knowlton RC, Elgavish RA, Limdi N, Bartolucci A, Ojha $\mathrm{B}$, Blount J, et al: Functional imaging: I. Relative predictive value of intracranial electroencephalography. Ann Neurol 64:25-34, 2008

19. Kwon HE, Eom S, Kang HC, Lee JS, Kim SH, Kim DS, et al: Surgical treatment of pediatric focal cortical dysplasia: clinical spectrum and surgical outcome. Neurology 87:945-951, 2016

20. Muthaffar O, Puka K, Rubinger L, Go C, Snead OC III, Rutka JT, et al: Reoperation after failed resective epilepsy surgery in children. J Neurosurg Pediatr 20:134-140, 2017

21. Najm IM, Tassi L, Sarnat HB, Holthausen H, Russo GL: Epilepsies associated with focal cortical dysplasias (FCDs). Acta Neuropathol 128:5-19, 2014

22. Noe K, Sulc V, Wong-Kisiel L, Wirrell E, Van Gompel JJ, Wetjen N, et al: Long-term outcomes after nonlesional extratemporal lobe epilepsy surgery. JAMA Neurol 70:10031008, 2013

23. O'Brien TJ, So EL, Mullan BP, Hauser MF, Brinkmann BH, Bohnen NI, et al: Subtraction ictal SPECT co-registered to MRI improves clinical usefulness of SPECT in localizing the surgical seizure focus. Neurology 50:445-454, 1998

24. Phi JH, Cho BK, Wang KC, Lee JY, Hwang YS, Kim KJ, et al: Longitudinal analyses of the surgical outcomes of pediatric epilepsy patients with focal cortical dysplasia. J Neurosurg Pediatr 6:49-56, 2010

25. Pomata HB, González R, Bartuluchi M, Petre CA, Ciraolo C, Caraballo R, et al: Extratemporal epilepsy in children: candidate selection and surgical treatment. Childs Nerv Syst 16:842-850, 2000

26. Ryvlin P, Rheims S: Predicting epilepsy surgery outcome. Curr Opin Neurol 29:182-188, 2016

27. So EL: Integration of EEG, MRI, and SPECT in localizing the seizure focus for epilepsy surgery. Epilepsia 41 (Suppl 3):S48-S54, 2000

28. Spencer SS, Berg AT, Vickrey BG, Sperling MR, Bazil CW, Shinnar S, et al: Predicting long-term seizure outcome after resective epilepsy surgery: the multicenter study. Neurology 65:912-918, 2005

29. Tassi L, Garbelli R, Colombo N, Bramerio M, Russo GL, Mai R, et al: Electroclinical, MRI and surgical outcomes in 100 epileptic patients with type II FCD. Epileptic Disord 14:257-266, 2012

30. Téllez-Zenteno JF, Dhar R, Wiebe S: Long-term seizure outcomes following epilepsy surgery: a systematic review and meta-analysis. Brain 128:1188-1198, 2005

31. Vickrey BG, Hays RD, Rausch R, Engel J Jr, Visscher BR, Ary CM, et al: Outcomes in 248 patients who had diagnostic evaluations for epilepsy surgery. Lancet 346:1445-1449, 1995

32. West S, Nolan SJ, Newton R: Surgery for epilepsy: a systematic review of current evidence. Epileptic Disord 18:113121,2016

33. Yao K, Mei X, Liu X, Duan Z, Liu C, Bian Y, et al: Clinical characteristics, pathological features and surgical outcomes 
of focal cortical dysplasia (FCD) type II: correlation with pathological subtypes. Neurol Sci 35:1519-1526, 2014

\section{Disclosures}

The authors report no conflict of interest concerning the materials or methods used in this study or the findings specified in this paper.

\section{Author Contributions}

Conception and design: Kankirawatana, Kim. Acquisition of data: Blount, Kankirawatana, Mohammed, Lauer, Kim, Harrison, Rozzelle, Knowlton. Analysis and interpretation of data: Blount,
Mohammed, Lauer, Li, Harrison, Goyal, Rozzelle, Knowlton. Drafting the article: Kankirawatana. Critically revising the article: Blount, Kankirawatana, Mohammed, Lauer. Approved the final version of the manuscript on behalf of all authors: Blount. Statistical analysis: Aban.

\section{Correspondence}

Jeffrey P. Blount: Children's of Alabama/UAB, Birmingham, AL. jeffrey.blount@childrensal.org. 